\title{
Artículos
}

\section{Migraciones y distribución espacial: migrantes sudamericanos en la Región Metropolitana de Buenos Aires, 2001-2010}

\section{Migrations and spatial distribution: South American migrants in the Metropolitan Buenos Aires, 2001-2010}

\author{
Albano Blas Vergara Parra*
}

\begin{abstract}
Resumen
El artículo se propone realizar un aporte al conocimiento de los patrones de asentamiento y distribución espacial de los inmigrantes internacionales, en particular de los colectivos migrantes de Sudamérica más dinámicos en la Región Metropolitana de Buenos Aires durante la década del 2000, a partir de un análisis estadísticocartográfico que utiliza como principal fuente de información los censos nacionales de población de 2001 y 2010. En el estudio se pone en evidencia la existencia de fuertes diferencias en la dinámica demográfica de los colectivos de Bolivia, Paraguay y Perú en relación con los restantes grupos de inmigrantes internacionales. Estos tres colectivos migratorios han explicado en gran medida los flujos de inmigración externa hacia dicha región metropolitana en las últimas décadas. Asi también, el trabajo muestra que la distribución espacial de tipo concentrado-segregado de estos grupos no sólo no se modificó, sino que se profundizó y reforzó aún más durante el periodo de análisis.
\end{abstract}

Palabras clave: Región Metropolitana de Buenos Aires; migración internacional; distribución espacial.

\begin{abstract}
This article purposes to contribute to the knowledge of the patterns of settlement and spatial distribution of international immigrants, particularly the most dynamic migratory South American groups in the Buenos Aires Metropolis during the decade of

* Universidad de Buenos Aires, Facultad de Ciencias Económicas, Instituto Interdisciplinario de Economía Política de Buenos Aires. Dirección postal: Córdoba 2122, 2 piso, 1120 AAQ, Buenos Aires, Argentina. Correo electrónico: albano.vergara@gmail.com
\end{abstract}


2000, based on an analysis in which the national population census of 2001 and 2010 are used as the main sources of information. The article shows the differences between the immigrant's groups from Bolivia, Paraguay and Peru and the other international immigrant groups, in relation to their demographic dynamics, through the period 2001-2010. These three migratory groups have largely explained the flows of external immigration that occurred in the last decade in the metropolitan region. Also, the work shows that the concentrated-segregated spatial distribution of these groups not only did not change, but also deepened and reinforced even more during the analysis period.

Keywords: Buenos Aires City; international migration; spatial distribution.

\section{Introducción}

Durante las últimas décadas del siglo XX, los colectivos de migrantes sudamericanos dirigidos hacia la Región Metropolitana de Buenos Aires, ${ }^{1}$ en particular los procedentes de Bolivia, Paraguay y Perú, han destacado por presentar gran dinamismo. Una de las particularidades de estos tres colectivos radica en su tipo de inserción laboral en determinados segmentos del mercado de trabajo (en específico, construcción, servicio doméstico y algunas industrias particulares y puestos de trabajo no cubiertos por la población nativa a causa de los bajos salarios y las malas condiciones de trabajo). Por otra parte, estos tres colectivos presentan también como característica una distribución de tipo concentrado-segregado en el espacio urbano de la región metropolitana.

En Argentina, el primer decenio del siglo XXI se caracterizó por un fuerte proceso de recuperación económica y expansión del empleo. En esta dirección, cabe decir que los periodos de mayor inmigración siempre estuvieron vinculados con fuertes aumentos en la demanda de mano de obra (Marshall, 1983). Por consiguiente, el análisis de los flujos migratorios hacia la región metropolitana de estos tres colectivos debe inscribirse en el marco del tipo de inserción laboral selectivo que éstos presentan, así como en el contexto macroeconómico de crecimiento intenso registrado entre 2001 y 2010.

El presente artículo se propone, como objetivo general, dar cuenta del comportamiento demográfico y la distribución espacial de los colectivos más dinámicos de inmigrantes sudamericanos de la Región Metropolitana de

1 La Región Metropolitana de Buenos Aires comprende a la división administrativa de la ciudad de Buenos Aires - capital de la República Argentina-, más un conjunto restante de treinta jurisdicciones-municipios que conforman la Aglomeración Gran Buenos Aires, en continua expansión. Según el censo de 2010, en la región habitan 13590219 personas, había 4306069 hogares, y la aglomeración comprende una superficie de $5075 \mathrm{~km}^{2}$. 
Buenos Aires (RMBA) durante 2001-2010, a partir de un análisis estadístico y cartográfico en el que se utilizan como principales fuentes de información los censos nacionales de población de 2001 y 2010. En este marco, se analiza la dinámica demográfica y la distribución territorial de los inmigrantes de Bolivia, Paraguay y Perú mediante un análisis espacial que procura establecer cuáles son sus principales características, especificidades y diferencias con los restantes grupos de inmigrantes internacionales. Por otro lado, el estudio analiza también si se produjeron cambios en los patrones de asentamiento de los migrantes de Bolivia, Paraguay y Perú, o si, por el contrario, éstos se han conservado. En este sentido, resulta pertinente proponer como hipótesis guía que, si estos tres colectivos durante el contexto previo al periodo de análisis ya destacaban por su fuerte dinamismo, sería esperable entonces que en un contexto de expansión económica y crecimiento de la demanda laboral (en particular en los segmentos en los que éstos se insertan) se haya producido un crecimiento extraordinario de los flujos migratorios de Bolivia, Paraguay y Perú en la región metropolitana, en detrimento de los restantes flujos externos.

El artículo se organiza del siguiente modo. En la primera sección se realiza una exposición de las principales características de los inmigrantes en Argentina, provenientes de los países limítrofes y de Perú. En la segunda, se explicitan las referencias teóricas y se realiza una revisión de los principales antecedentes empíricos. En la siguiente sección se especifican las características de las fuentes de información utilizadas (censos nacionales de población), así como la metodología aplicada en el análisis estadístico-cartográfico llevado a cabo. En la cuarta sección se presentan los principales hallazgos obtenidos en el estudio y, por último, se proponen algunas conclusiones e interpretaciones con base en los análisis y resultados alcanzados.

\section{Especificidades de los migrantes de países limítrofes y de Perú en Argentina}

Los movimientos de la población provenientes de países limítrofes (Chile, Bolivia, Paraguay, Brasil y Uruguay) hacia Argentina tienen una larga tradición. Estas poblaciones han presentado históricamente dos grandes rasgos en cuanto a sus patrones de asentamiento territorial (Cerrutti, 2009): uno caracterizado por una significativa concentración en la metrópoli de Buenos Aires de inmigrantes uruguayos, brasileños y paraguayos, y otro caracterizado por una mayor dispersión geográfica a lo largo del territorio entre inmigrantes chilenos y bolivianos. Históricamente, estos flujos migratorios 
hacia Argentina se produjeron en zonas transfronterizas caracterizadas por compartir similares modalidades económicas, sociales y culturales (Maguid, 2008). De este modo, hasta casi la mitad del siglo XX, las provincias fronterizas fueron el principal lugar de destino. Ahora bien, a partir de 1960 la migración de países limítrofes comenzó a direccionarse cada vez más hacia la metrópoli de Buenos Aires, convirtiéndose ésta en el principal lugar de destino. En este sentido, la metropolización del sistema migratorio en Argentina y el papel centralizador que adquirió la RMBA -no sólo en cuanto a la concentración de población, sino también de capitales, inversiones y comercio- sugieren la pertinencia de concebir a Buenos Aires como una ciudad global para interpretar y analizar los fenómenos migratorios (Mármora, 2002). Es así que, para mediados de la década de 1980, la metrópoli de Buenos Aires ya se había convertido en un subsistema migratorio de relevancia dentro de la región del Cono Sur (Balán, 1990).

Durante el periodo 1960-1990, la inmigración de países limítrofes incrementó su peso en el total de la población inmigrante de la RMBA, pasando del 25 al 47\%, a pesar del creciente deterioro que la economía argentina comenzó a registrar hacia finales de la década de 1970 (Maguid y Arruñada, 2005). Durante este periodo, del conjunto de países limítrofes, la participación -según orden de importancia-se conformaba por Paraguay, Uruguay, Bolivia, Chile y Brasil (Maguid y Bruno 2010). Es importante señalar que la inmigración de Perú en la región metropolitana en esta etapa no era significativa.

A partir de la década de 1980 comenzó a registrarse un fuerte crecimiento de los colectivos inmigratorios de Paraguay y Bolivia, que se tradujo en el mayor peso relativo que adquirió cada una de estas colectividades en el conjunto de inmigrantes internacionales. Más adelante, a partir de la década de 1990 comenzó a consolidarse una "nueva corriente migratoria" proveniente de Perú (Cerruti, 2005; Rosas, 2010a). Durante dicha década se produjo un fuerte cambio de composición en el conjunto de la población inmigrante, en el que destacaron los paraguayos, bolivianos y peruanos por ser los colectivos con el mayor dinamismo en la RMBA.

En el caso de la inmigración boliviana en el territorio argentino, ésta adquirió gran significación hacia mediados del siglo XX y mantuvo una tendencia ascendente que se intensificó durante la década de 1990 (Benencia, 2003). En este sentido, Sassone y Demarco (1994) distinguen cuatro etapas de la inmigración boliviana en Argentina que reflejan la alta movilidad y adaptabilidad que presentó este grupo para redistribuirse territorialmente en función de la demanda de empleo, que son: 1) 1920-1950, etapa caracterizada por la expansión de la producción azucarera en las provincias limítrofes de Jujuy y Salta, que favorecieron la afluencia de trabajadores rurales; 
2) 1950-1960, etapa caracterizada por la complementación de dichas actividades con el trabajo en el tabaco y la frutihorticultura;23) 1960-1970, periodo en que se incrementa la presencia de bolivianos/as en Buenos Aires, Mendoza y, en menor medida, en Córdoba, y al mismo tiempo se atenúa la presencia en las provincias limítrofes; 4) 1970-2000, periodo en que comienza a darse una mayor dispersión territorial y difusión ocupacional. Hacia fines de la década de 1980 se acentúa la propensión de este grupo a dirigirse hacia la RMBA. De este modo, a comienzos de la década de 1990 el 39\% del total de bolivianos que residían en Argentina se localizó en la RMBA, cifra que se elevó al 52\% para fines de dicha década. Para el año 2001, uno de cada dos inmigrantes bolivianos/as que residían en Argentina se encontraba en la metrópoli de Buenos Aires.

Por su parte, la inmigración paraguaya para finales del siglo XIX ya registraba una fuerte presencia en el territorio argentino, principalmente en las provincias limítrofes de Misiones, Corrientes y Formosa (sólo una minoría residía en Buenos Aires). Así, la inmigración paraguaya, hasta mediados del siglo XX, se orientaba principalmente a abastecer los requerimientos de mano de obra estacional de la industria forestal y de la producción de algodón, caña de azúcar, tabaco y yerba mate de las provincias limítrofes (Meichtry y Beck, 2002). Durante este periodo, la fuerza de trabajo paraguaya comenzaba a ser requerida como sustituto de la mano de obra nativa, que se desplazaba hacia los grandes centros urbanos provinciales, especialmente Buenos Aires. Con el auge de la industrialización sustitutiva, durante el periodo de 1947-1970 se produjo un fuerte incremento del flujo migratorio, y este colectivo pasó a convertirse en el más importante dentro del grupo de los países limítrofes. Ahora bien, en la década de 1980 el número de paraguayos/as que residían en Argentina se redujo, y este movimiento expresó no sólo un cese del flujo sino también - posiblemente- un moderado proceso de retorno (Cerrutti, 2009). ${ }^{3}$ Con la implementación del Plan de Convertibi-

2 Según el censo de 1947, del total de bolivianos/as que residían en Argentina, el 88\% se encontraba en las provincias de Salta y Jujuy, y sólo 8\% en la RMBA (Sassone y Demarco, 1994).

Según la autora, esto en parte pudo haberse debido a los efectos de la llamada "década perdida" en Argentina. Durante los años de 1980, Paraguay logró mantener su PIB per cápita; sin embargo, en los años subsiguientes la economía paraguaya se estancó, mientras que, por el contrario, el PIB per cápita argentino creció. En este sentido, la migración paraguaya ha mostrado ser sensible a los cambios macroeconómicos relativos entre ambos países. Parrado y Cerrutti (2003) encontraron que la probabilidad de emigrar a Argentina por parte de los varones paraguayos no sólo se asocia a una serie de rasgos individuales, sino que también se ve afectada por la relación entre la situación económica relativa de Paraguay y Argentina. En concreto, se encontró que a mayor diferencia entre el PIB per cápita argentino relativo al paraguayo se incrementa la probabilidad de emigrar hacia Argentina. 
lidad en el año 1991 (paridad de la moneda local con la divisa internacional), Argentina se convirtió, sin lugar a duda, en uno de los principales centros de atracción y lugar de destino. En este contexto, durante la década de 1990 la inmigración paraguaya volvió a intensificarse y el stock de inmigrantes procedentes del mismo país volvió a crecer de modo intenso.

Por último, la inmigración peruana presenta una serie de peculiaridades puesto que su presencia en el territorio argentino -como se mencionó- fue poco significativa en términos históricos, y más aún su presencia en la metrópoli de Buenos Aires (Rosas, 2010a). Sin embargo, a partir de la década de 1980, y principalmente durante la siguiente, Argentina se constituyó en uno de los principales destinos migratorios del llamado "éxodo peruano". La delicada situación económico-social del país y, en particular, la espiral creciente de violencia política ocurrida durante los años ochenta, fueron dos aspectos clave de la masiva emigración ocurrida en Perú (Cerrutti, 2005). En ese sentido, el flujo de inmigrantes de origen peruano hacia Argentina durante la década de los noventa resultó muy significativo, convirtiéndose quizás en uno de los casos más sobresalientes de los últimos decenios en cuanto a la inmigración regional (Rosas, 2010a). A continuación, se explicitan -de modo breve- las referencias teóricas del estudio y se realiza una revisión de los principales antecedentes empíricos.

\section{Referencias teóricas y antecedentes empíricos}

Las investigaciones sobre la distribución espacial de los inmigrantes en las ciudades receptoras tienen una extensa tradición. A comienzos del siglo XX, en el marco de los intensos flujos de migración hacia las principales ciudades de Estados Unidos, de la creciente segregación espacial de las minorías étnicas y de la preocupación de las clases dominantes por su integración, emergió la Escuela de Chicago y la denominada "ecología humana" (Burgees, 1925; McKenzie, 1925; Park, 1926), de la que surgieron numerosos trabajos. A partir de sus principales lineamientos, los estudios enmarcados en esta corriente interpretaron las pautas residenciales de los migrantes a partir de dos grandes modelos explicativos (Darroch y Marston, 1971). Uno es el denominado modelo económico derivado de la hipótesis ecológica de la asimilación-assimilation hypothesis-; ${ }^{4}$ su argumento principal sostiene que

4 La hipótesis clásica de la asimilación espacial postula -a grandes rasgos- que los migrantes recientes tienden a emplazarse en la ciudad de forma concentrada-segregada en enclaves étnicos y, a medida que se desarrolla el proceso de aculturación en tales grupos, progresivamente se van asimilando en la sociedad receptora y dispersando espacialmente en la ciudad. 
en las diferencias socioeconómicas se encuentran las bases de la segregación. El segundo modelo, denominado étnico, sostiene, por el contrario, que el principal determinante es de orden psicosocial.

En este sentido, es necesario señalar que segregación no es equivalente a concentración. Es decir, la concentración espacial de los distintos grupos en la ciudad no significa necesariamente segregación (Linares, 2013). Asimismo, es conveniente precisar los alcances del concepto de segregación, puesto que éstos dependen de su anclaje teórico. Desde la perspectiva teórica de la ecología humana, las distancias espaciales son entendidas como distancias sociales producto de lógicas individuales. Por tanto, la segregación es producto de las diferencias en las capacidades económicas, de elección y apropiación del espacio urbano que tienen los grupos y los individuos. Ahora bien, desde la perspectiva de la geografía social crítica, la segregación se entiende como producto de la organización del espacio urbano en unidades con una gran homogeneidad social interna y con una disparidad social entre ellas (Castells, 1979; Harvey, 1997). Estas disparidades tienden a perpetuarse ya que condicionan en forma duradera la vida de los individuos, dado que la segregación espacial implica necesariamente una distribución desigual de los recursos y las oportunidades. ${ }^{5}$ Por otra parte, cabe agregar que para el análisis y la comprensión de los patrones de asentamiento espacial de los grupos migratorios, la literatura señala tres aspectos relevantes y conectados entre sí. Éstos son: el capital social ${ }^{6}$ (redes sociales), las oportunidades laborales y las oportunidades de alojamiento de los lugares de destino (Leekers, Engbersen y San, 2007). De estas dimensiones, el capital social con el que cuentan los migrantes tiene gran relevancia, puesto que resulta un facilitador de oportunidades laborales y de alojamientos. En este sentido, los nuevos migrantes tienden a asentarse en las áreas urbanas en las que otros connacionales ya se asentaron previamente.

El proceso secuencial implica que los grupos inicialmente se concentran en áreas específicas, y luego mediante la fusión con los patrones culturales - principalmente en las sucesivas generaciones-, se produce un proceso de dispersión en el espacio residencial-urbano. Cabe señalar que estos postulados han sido objeto de varias críticas y objeciones, en particular porque los modelos explicativos tuvieron como principal centro de gravitación el contexto histórico-social de Estados Unidos.

5 La segregación conforma una categoría clave para comprender la estructuración del espacio intraurbano en las metrópolis, así como también para entender la distribución espacial de los distintos grupos sociales, no sólo como un simple reflejo de los procesos sociales, sino también como un condicionante de éstos (Villaça, 1998).

6 Desde la propuesta de Portes (1998), por capital social se hace referencia a las capacidades de los migrantes para movilizar recursos -tales como dinero, trabajo, alojamiento, información-mediante redes familiares o de amistades. 
En el contexto de América Latina, varias investigaciones recientes sobre segregación espacial han coincidido en señalar la presencia de este fenómeno en sus principales metrópolis. En este marco, destaca el trabajo de Rodríguez y Arriagada (2004) en el que se muestra que las principales áreas metropolitanas de la región son ciudades segregadas. Ahora bien, los autores indican también que su intensidad no alcanza los niveles de segregación étnico-raciales que presentan las ciudades de Estados Unidos. En este sentido, los autores precisan que los niveles de segregación de los grupos migratorios en Latinoamérica no son elevados, sino que más bien este fenómeno se produce entre los grupos socioeconómicos polares cuyos grados de segregación son muy intensos, tendencia que se ha ido profundizado durante las últimas décadas. ${ }^{7}$

En el contexto de Argentina, las investigaciones sobre distribución espacial de migrantes son múltiples. Algunas de ellas provienen de los estudios sociohistóricos, como el de Otero y Pellegrino (2004), sobre pautas residenciales de migrantes en las ciudades de Buenos Aires y Montevideo durante la inmigración masiva. Otra gran parte remite a investigaciones provenientes de la geografía social, orientadas a diversos grupos migratorios, en las que se estudian sus patrones de asentamiento a través del análisis socioespacial en distintas ciudades argentinas. ${ }^{8}$ Por otra parte, cabe decir que gran cantidad de estudios recientes han comenzado a incorporar al análisis factores tales como las estrategias de localización, las redes sociales, la dinámica del mercado inmobiliario y las prácticas discriminatorias sobre ciertos grupos migratorios, entre otros. No obstante, según Marcos y Mera (2015a), en Argentina las investigaciones con abordajes de tipo cuantitativo son actualmente más bien escasas.

En particular, entre los trabajos previos sobre migrantes de países limítrofes con Argentina destaca el de Molinatti y Peláez (2014) en el que se

7 El estudio que llevan a cabo Rodríguez y Arriagada (2004) sobre las pautas de localización de grupos socioeconómicos polares mediante microdatos censales de Chile (Área Metropolitana del Gran Santiago, 1992), México (Zona Metropolitana de la Ciudad de México, 2000) y Perú (Área Metropolitana de Lima, 1993), muestra que las elites con altos ingresos en estos tres centros urbanos presentan un claro patrón de concentración territorial, bajo una modalidad de segregación residencial socioeconómica (SRS) a gran escala. El trabajo destaca que la SRS en Santiago de Chile es particularmente intensa puesto que las áreas ocupadas por la elite son altamente homogéneas.

8 Entre éstos destacan los aportes de Perren (2010) para la Ciudad de Neuquén; los estudios realizados por Sassone y De Marco (1994) y por Sassone, González y Matossian (2010) sobre las pautas de asentamiento de los inmigrantes limítrofes en Argentina; el análisis de Buzai, Baxendale, Rodríguez y Escanes (2003) sobre la ciudad de Luján; el trabajo de Mignone (2010) respecto a la segregación residencial de los paraguayos en las ciudades de Formosa y Posadas; y el trabajo de Matossian (2010) sobre la segregación espacial de los chilenos en Bariloche, entre otros. 
utilizan herramientas de estadística espacial para analizar la segregación residencial de migrantes peruanos y bolivianos en la ciudad de Córdoba, en los años 2001 y 2008. ${ }^{9}$ Otro antecedente relevante es la investigación de Marcos y Mera (2015a) en la que se analiza la distribución espacial de todos los grupos migratorios en la Aglomeración Gran Buenos Aires durante el año 2001. En este estudio, las autoras sostienen que en la distribución territorial de los distintos grupos de inmigrantes internacionales se puede trazar un "continuum" que va desde colectivos relativamente equis-distribuidos (como el caso de los uruguayos) hasta colectivos que han tendido a residir en zonas muy específicas y diferenciadas (como el caso de los coreanos). Asimismo, existen otros colectivos cuya localización espacial coincide con las proximidades de los centros administrativos y de negocios (por ejemplo, el corredor geográfico norte, en el caso de colombianos y estadounidenses); $\mathrm{y}$, por otro lado, colectivos como los de los paraguayos, los bolivianos y los peruanos, que se localizan de modo concentrado en zonas caracterizadas por déficits de servicios e infraestructura. Con respecto a las especificidades en los patrones de localización de los migrantes paraguayos y bolivianos en la ciudad de Buenos Aires, Marcos y Mera (2015b) señalan que éstos tienen como principal alternativa de alojamiento los hábitats informales (conocidos como villas de emergencia y asentamientos precarios). Por su parte, si bien una importante proporción de migrantes peruanos se localiza en hábitats informales, un gran porcentaje se ha asentado en áreas residenciales de sectores medios.

En resumen, los migrantes de Bolivia, Paraguay y Perú han tendido a concentrarse en áreas geográficas específicas de la RMBA, mayormente en los espacios habitacionales deficientes y, aún más, entre quienes arribaron de manera reciente. En este marco, Maguid y Bruno (2010) consideran que la existencia de mayores condicionamientos por parte de la mano de obra proveniente de dichos países para aceptar condiciones laborales precarias y remuneraciones más bajas en relación con sus pares nativos, ha tendido a facilitar su rápida inserción laboral (incluso en periodos de alta desocupación). En consecuencia, las condiciones de inserción laboral de los migrantes de Bolivia, Paraguay y Perú tienen su correlato en las condiciones

9 En otro interesante trabajo sobre los patrones de asentamiento de los peruanos en la ciudad de Córdoba en el año 2001, de Gómez y Sánchez Soria (2017), se sugiere que si bien los resultados obtenidos se ajustan a un proceso coherente con la teoría clásica de la asimilación (fusión con la sociedad receptora, dispersión residencial progresiva), varios elementos no apoyarían esta interpretación de modo lineal. En este sentido, los autores proponen una hipótesis alternativa en la que la composición de clase y la temporalidad de los flujos de migrantes peruanos habrían articulado distintas modalidades de incorporación e inserción espacial en la ciudad receptora.

Estudios Demográficos y Urbanos, vol. 34, núm. 2 (101), 2019, pp. 301-335 doi: http://dx.doi.org/10.24201/edu.v34i2.1800 
habitacionales que estos colectivos presentan. En este sentido, tal como sostiene Cerruti (2009), los colectivos migratorios que más crecieron en la región metropolitana fueron también los que más segregados se encuentran espacialmente. A continuación, se presenta la metodología y las fuentes de información utilizadas en el estudio.

\section{Fuentes de información y metodología utilizada}

En primer lugar, existen numerosas fuentes de información que posibilitan adentrarse en el estudio de las migraciones, aunque sin lugar a duda las más utilizadas por los especialistas son los censos de población; éstos cuentan con alta cobertura, alcance y regularidad, y posibilitan comparar datos entre distintos países y momentos (Cerruti, 2009). Mediante dicha fuente de información es posible analizar la dinámica de los distintos stocks poblacionales, así como también describir y caracterizar a los contingentes migratorios a partir de un conjunto muy amplio de dimensiones de análisis. Sin embargo, así como los censos de población presentan enormes ventajas, cuentan también con un conjunto de limitaciones, entre las que destaca la periodicidad, es decir, su lapso de levantamiento decenal dificulta el estudio de las tendencias de corto plazo. Otra limitante es que, debido a que los censos de población no tienen como objetivo principal el análisis de la migración, la información que recopilan sobre este tema suele ser bastante reducida. Dado que una de las características más relevantes del estudio de la migración es su temporalidad (es decir, el momento de partida y llegada), la escasez de este tipo de información resulta una restricción muy importante. Ahora bien, más allá de estas limitaciones, los censos de población continúan siendo una de las fuentes más confiables para estimar el tamaño, la evolución y las características de los inmigrantes.

Para el presente artículo se utilizó como fuente de información los censos nacionales de población de 2001 y 2010 del Instituto Nacional de Estadísticas y Censos (INDEC). Cabe señalar que, a partir del operativo censal de 2001, el INDEC comenzó a publicar la información censal en formato digital (microdatos) mediante un nuevo sistema informático (Redatam) que posibilita el desagregamiento de la información en diferentes escalas espaciales de análisis. De este modo, la posibilidad de georreferenciar la información censal de 2001 y 2010 resultó una herramienta de vital importancia para el análisis que se llevó a cabo en el presente estudio.

En segundo lugar, para el recorte espacial de la Región Metropolitana se tomó la definición utilizada por Alfredo Garay (2007), esto es: "Ciudad 
de Buenos Aires más un conjunto de treinta partidos-municipios pertenecientes a la conurbación bonaerense que conforman una envolvente de tres cordones o coronas. ${ }^{10}$ En este marco, los análisis espaciales efectuados utilizaron dos recortes geográficos que surgen como formas distintas de agregación espacial de datos para los que se dispone información en las fuentes censales. El primero se conforma por las principales unidades que presenta la región metropolitana: esto es, la ciudad central (ciudad de Buenos Aires) más sus tres envolventes (primera, segunda y tercera corona de la conurbación). El segundo por los corredores geográficos Norte, Oeste y Sur de la Región Metropolitana (Mapa 1). Así, con base en la superposición de ambos recortes espaciales, se subdividió el territorio en 16 zonas intrametropolitanas (Mapa 2). En el caso de la conurbación, se delimitaron nueve zonas a partir de la división de las envolventes según los corredores geográficos. En el caso de la ciudad de Buenos Aires se aplicó el mismo criterio y se añadió - dada la densidad poblacional de su territorio- un criterio adicional. Por consiguiente, se estableció primero la zona Centro y luego se delimitaron seis zonas producto de la subdivisión de los tres corredores geográficos. Cabe señalar que el uso de estos últimos se funda en la relevancia y la fuerte relación que tienen los corredores geográficos con las características socioeconómicas de la estructura urbana de la metrópoli de Buenos Aires (Schteingart y Torres, 1973). ${ }^{11}$

En tercer lugar, es necesario precisar que el análisis de la distribución espacial y la evolución de los grupos migrantes se llevó a cabo mediante el nivel de hogares (jefes/as). Esta decisión se fundamentó a partir de dos criterios. El primero, fundado en el supuesto de que la unidad de análisis hogar

10 La RMBA ha sido subdividida por estudiosos y planificadores en cordones o coronas. Para su delimitación se considera -además de criterios de carácter socioeconómico- la mayor o menor cercanía con respecto a la ciudad de Buenos Aires, dibujando periferias concéntricas en torno a ésta. De este modo, gran parte de la literatura considera a los 24 municipios del Conurbano Bonaerense distribuidos en los dos primeros cordones o coronas, mientras que al resto de los municipios en una tercera corona.

11 En el estudio sobre la metropolización de Buenos Aires, Schteingart y Torres (1973) analizan este proceso a partir del establecimiento de grandes periodos históricos con el objeto de explicar las transformaciones ocurridas en la estructura urbana. A grandes rasgos, los autores sostienen que durante el periodo 1869-1930 (momento en el que se produjo la mayor expansión demográfica de la metrópoli) se produjeron dos procesos ecológicos residenciales de gran envergadura: uno basado en el desplazamiento de los grupos de altos ingresos hacia la zona Norte de la ciudad y otro basado en la ocupación de la zona Sur por parte de los inmigrantes de ultramar que conformaban el grueso de las filas del proletariado urbano. En el siguiente periodo (1930-1960) la estructura básica de la metrópoli fijada durante la primera etapa no se alteró en sus aspectos claves y, de este modo, se mantuvieron tres sectores principales estructurados en torno de ejes circulatorios (Norte, Oeste y Sur). En consecuencia, la distribución territorial de los grupos socioeconómicos en la metrópoli de Buenos Aires se encuentra muy asociada a sus corredores geográficos. 


\section{Mapa 1}

Región Metropolitana de Buenos Aires: coronas y corredores geográficos
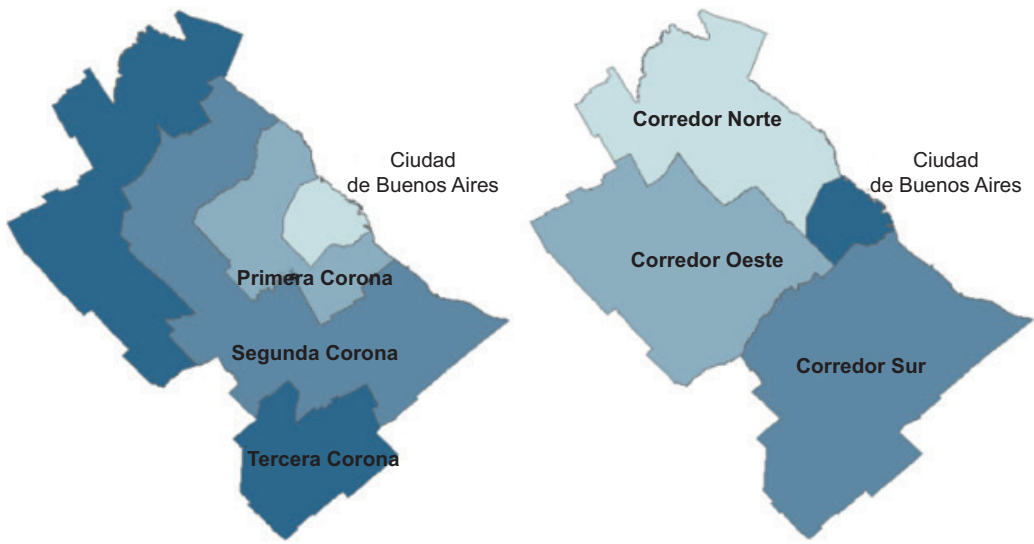

40

20

0

40 kilómetros

Fuente: Elaboración propia.

tiene una fuerte correspondencia con la unidad vivienda. En consecuencia, dado que el interés del estudio se encuentra en el análisis de los patrones de asentamiento, se consideró más apropiado el uso de la unidad hogares en vez de la unidad individuos. El segundo criterio está fundamentado en el supuesto de que el fuerte dinamismo del grupo migrante de Bolivia, Paraguay y Perú estuvo impulsado y enmarcado principalmente por el ciclo expansivo que generó una fuerte demanda laboral en los principales segmentos en los que se insertan estos tres colectivos. Así, dado que los jefes/as de hogar-principalmente en edades activas- tienen una mayor participación en el mercado laboral, y los restantes miembros del hogar constituyen -a priori- una fuerza de trabajo secundaria - en reserva-, se consideró más pertinente analizar la evolución a partir de dicha unidad de análisis.

En cuarto lugar, para el análisis de la migración se utilizó la variable país de nacimiento, clasificando los hogares en tres categorías: 1) hogares cuyos jefes/as nacieron en Argentina; 2) hogares cuyos jefes/as nacieron en Bolivia, Paraguay o Perú; y 3) hogares cuyos jefes/as nacieron en el extranjero, excluyendo los tres países citados. El criterio de separar los migrantes 


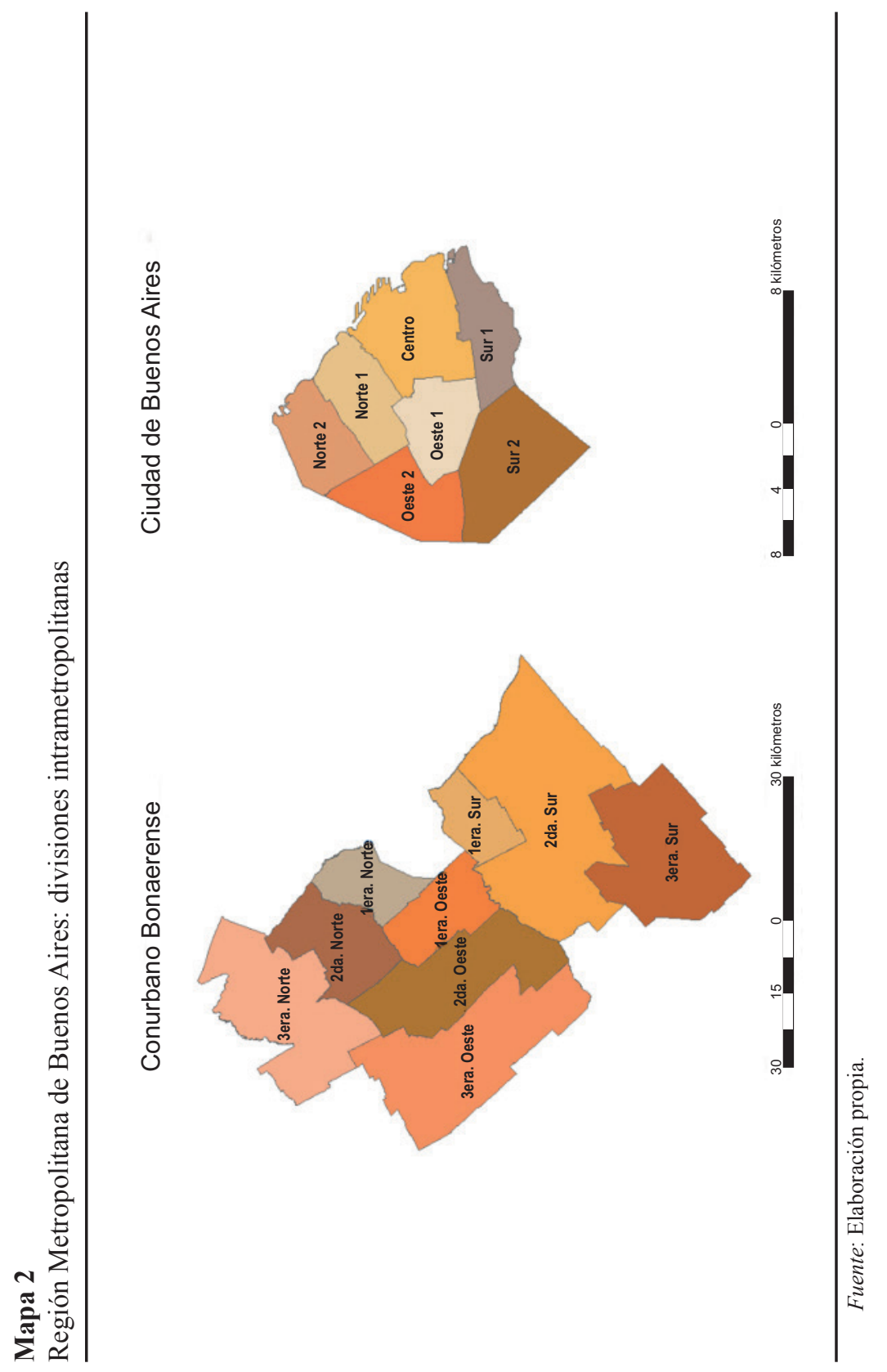


sudamericanos a partir de los tres países de origen mencionados responde a los principales interrogantes planteados. Asimismo, la decisión de utilizar como categoría de comparación al resto de los grupos migrantes se debe al tamaño que presentan los colectivos en el conjunto de la población inmigrante en la RMBA (en 2010, del total de hogares con jefes extranjeros, cerca de dos tercios son de Bolivia, Paraguay y Perú).

Por último, cabe señalar que debido a los cambios instrumentados en la metodología y en los cuestionarios del último censo de $2010,{ }^{12}$ no se pudieron utilizar indicadores de gran relevancia para el análisis demográficomigratorio. En concreto, no se dispone de la pregunta que discrimina la migración interna (es decir, a los nacidos en otras jurisdicciones y provincias) $\mathrm{y}$ tampoco de la pregunta que distingue a la migración reciente de la no reciente (es decir, quienes ingresaron al país en los últimos años). ${ }^{13}$ Este tipo de restricción de la fuente de información ha imposibilitado efectuar una serie de análisis de gran relevancia para el objeto de estudio que se abordó. A continuación, se presentan y analizan los principales resultados de la investigación.

\section{Patrones de asentamiento y cambios de composición demográfica}

En primer lugar, se presentan los análisis de la dinámica demográfica 20012010 de los inmigrantes internacionales en la RMBA, haciendo énfasis en las variaciones intra y entre grupos (en particular, en los inmigrantes limítrofes y de Perú). Asimismo, se analizan los comportamientos a nivel personas y hogares según los recortes espaciales señalados. En segundo lugar, se analiza la distribución espacial de los hogares con jefes/as nacidos en Bolivia, Paraguay y Perú y sus variaciones durante el periodo de análisis. Por último, se efectúa el mismo análisis para la población de los hogares con jefes extranjeros (excluyendo los tres países mencionados) con el objetivo de analizar sus comportamientos y diferencias durante el periodo.

12 En el operativo censal de 2010 se volvió a utilizar la metodología de censo con muestra, como en los casos de los censos de 1980 y 1991. De este modo, en las viviendas particulares se aplicó un cuestionario ampliado (A) para las unidades muestrales, y para las restantes un cuestionario básico (B). El cuestionario (A) fue utilizado en todas las localidades de menos de 50000 habitantes y en una muestra del 10\% de las viviendas ubicadas en las localidades de más de 50 000. En las restantes viviendas de las mismas localidades se utilizó el cuestionario (B) (INDEC, 2010).

13 La pregunta-indicador “¿En dónde residía hace cinco años?” en 2010 se formuló en el cuestionario ampliado (A) y, en consecuencia, no fue publicada en la base de microdatos (Redatam, INDEC) ya que sólo se incluyeron las preguntas del cuestionario básico (B). 


\subsection{Dinámica inmigratoria en RMBA. Periodo 2001-2010}

Según los datos censales, durante la década de 2000 se registró un claro pero pequeño crecimiento de inmigrantes internacionales en la RMBA. En términos de participación, éstos pasaron del 7.7\% en 2001 al 8.6\% en 2010, lo que, en términos absolutos, representó un incremento en el stock en cerca de 196 mil personas. Ahora bien, si se observa la evolución según la procedencia (país de nacimiento), se advierte un notable cambio de composición de la migración internacional en la región metropolitana, puesto que la población oriunda de países limítrofes y Perú registró un incremento del $50 \%$, mientras que la población proveniente de los restantes países del mundo se contrajo en 27\% (Cuadro 1). En términos absolutos, el primer grupo incrementó su stock en cerca de 300 mil personas, mientras que el segundo presentó una reducción de cerca de 105 mil.

Al analizar de modo específico la composición de la población inmigrante de países limítrofes y Perú, se puede apreciar que fueron los paraguayos/as quienes más aportaron al crecimiento y los que se mantuvieron en el primer lugar (Cuadro 2). En términos de participación, éstos pasaron del $42 \%$ en 2001 al 49\% en 2010. En otras palabras, en 2010 uno de cada dos inmigrantes en la RMBA desde países limítrofes y Perú proviene de Paraguay. El segundo grupo con mayor crecimiento, en términos relativos, fue el de los peruanos, que crecieron en el orden del $70 \%$. Sin embargo, en términos absolutos, fueron los bolivianos quienes se quedaron en segundo lugar, incrementando su stock en cerca de 67 mil personas, frente al incremento de 49 mil del grupo de peruanos.

Si se observa el crecimiento neto de la población ${ }^{14}$ de inmigrantes de países limítrofes y Perú en la RMBA, 61\% correspondió a Paraguay, 21\% a Bolivia y $16 \%$ a Perú. Asimismo, cabe destacar que durante el periodo se produjo una contracción de la población de Chile y Uruguay. Ahora bien, este movimiento debiera matizarse puesto que el stock de población se mantuvo relativamente estable; no obstante, dada la expansión que se produjo en los inmigrantes de Paraguay, Bolivia y Perú en RMBA, estos grupos -como contrapartida- perdieron peso en el conjunto. En efecto, en términos de participación, en 2001 los uruguayos ocupaban el tercer puesto y en 2010 pasaron a ocupar el cuarto, y en su lugar se ubicaron los inmigrantes peruanos (Cuadro 2).

14 El crecimiento neto de la población inmigrante surge como la diferencia entre el total de población en $\mathrm{t}_{2} \mathrm{yt}_{1}$. 


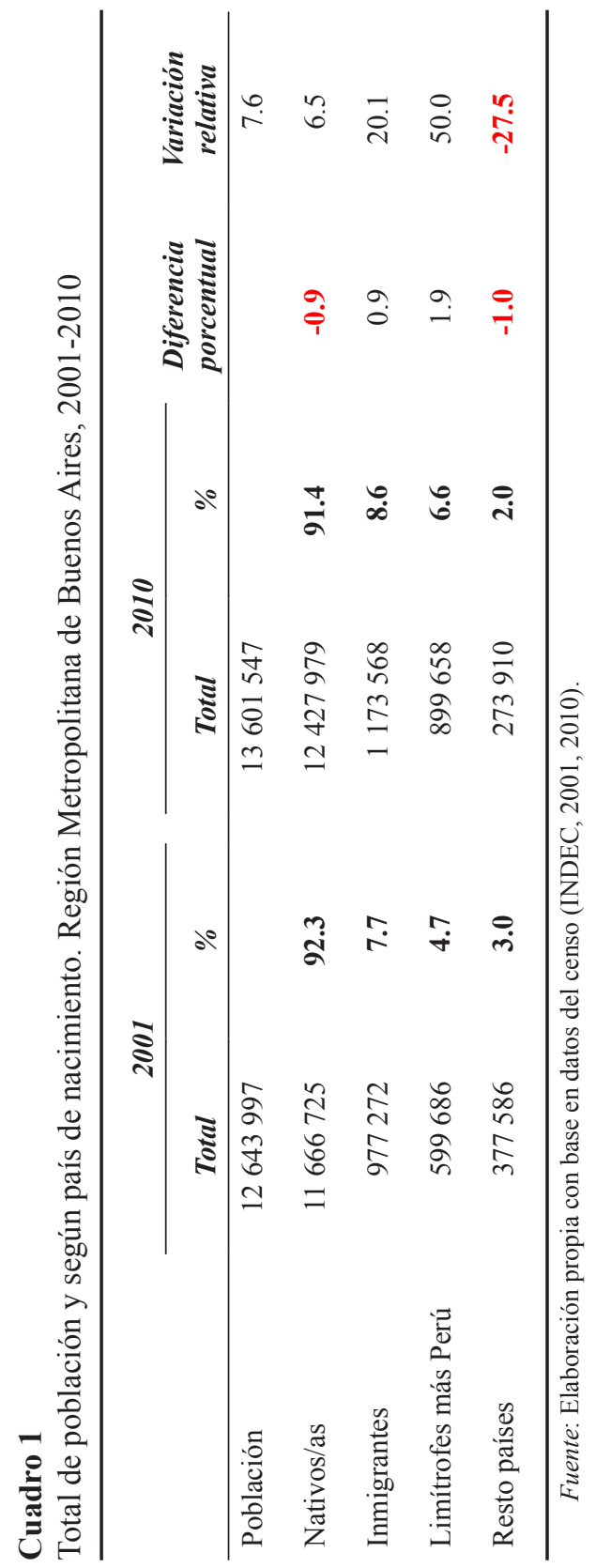




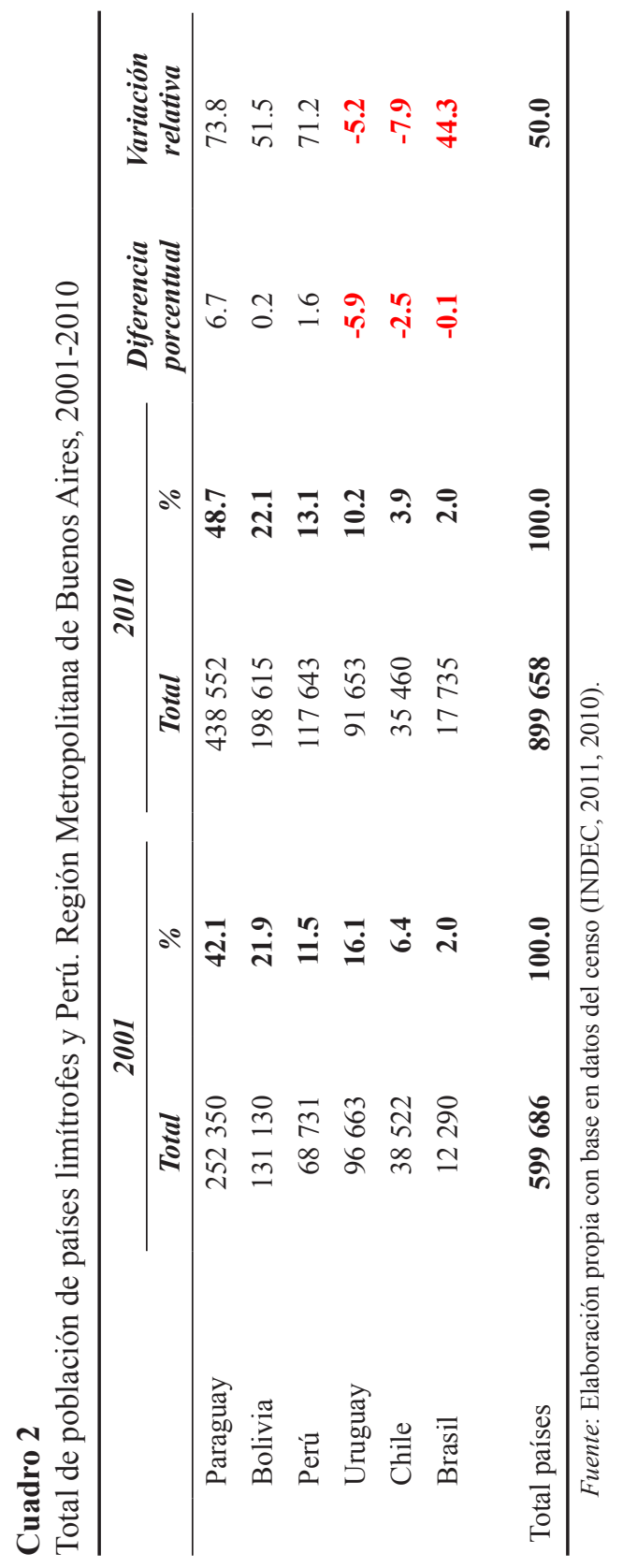


Si se analiza el comportamiento demográfico en la RMBA de los hogares, se advierte que durante el periodo se produjo un importante crecimiento en el que se pasó de 3.5 millones de hogares en 2001 a 4.3 millones de hogares aproximadamente en 2010 (que representó una variación relativa de $21.3 \%$ ). La composición de los hogares según la procedencia del jefe (país de nacimiento) no muestra cambios significativos: los hogares con jefes nacidos en el extranjero representaban el $11.9 \%$ del total en 2001 y el $11.4 \%$ en 2010 (Cuadro 3).

Ahora bien, el notable cambio de composición de la inmigración internacional, en particular de países limítrofes y Perú, observado a nivel personas en la RMBA, también mantuvo un correlato en el ámbito de los hogares. Durante el decenio, los hogares con jefes nacidos en países limítrofes y Perú tuvieron un incremento relativo de 49\% (115 mil hogares más), mientras que los hogares nacidos en otros países se contrajeron en 25\% (48 mil hogares menos). De este modo, se puede afirmar que la composición de hogares con jefes extranjeros ha experimentado un fuerte cambio entre 2001 y 2010. Los hogares con jefes de Bolivia, Paraguay y Perú pasaron de representar el $38 \%$ del total en 2001 al 57\% en 2010 (Gráfica 1).

\section{Gráfica 1}

Proporción de hogares con jefatura de Bolivia, Paraguay y Perú con relación al total de hogares con jefatura extranjera. Región Metropolitana de Buenos Aires, 2001-2010

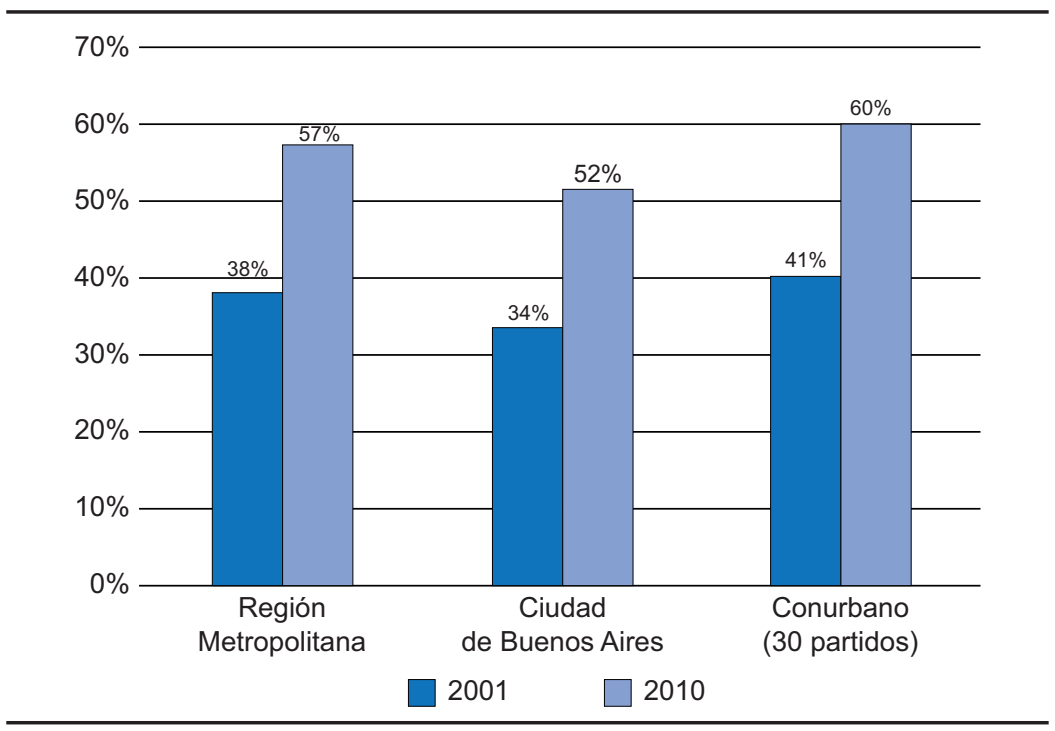

Fuente: Elaboración propia con base en datos del censo (INDEC, 2001, 2010). 


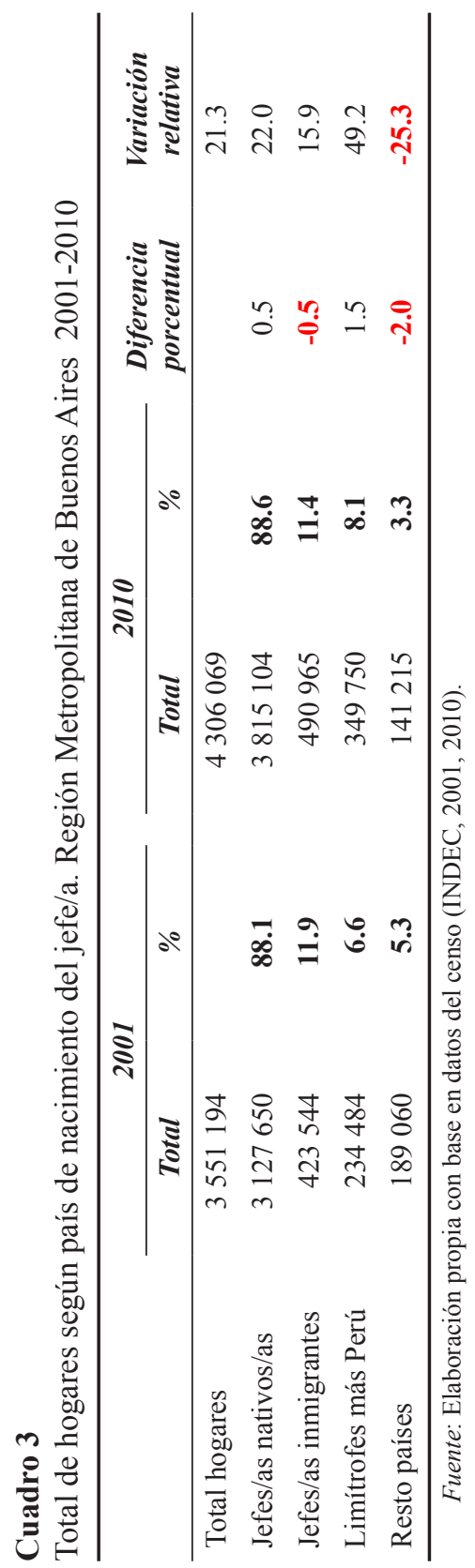


Este fuerte cambio de composición se ha expresado a nivel territorial tanto en la ciudad de Buenos Aires como en el Conurbano Bonaerense. En esta dirección, se advierten claras diferencias en la región metropolitana según cuáles sean las divisiones geográficas que se consideren. En el Conurbano, en particular en la Segunda Corona, los hogares con jefes nacidos en Bolivia, Paraguay y Perú pasaron de 52\% en 2001 a 69\% en 2010 (Gráfica 2). En este territorio, en el año 2010 cerca de siete de cada diez hogares con jefatura extranjera procedían de dichos países.

\subsection{Distribución espacial de los hogares con jefatura nacida en Bolivia, Paraguay y Perú}

Ya vimos que los hogares con jefes nacidos en Bolivia, Paraguay y Perú se incrementaron durante el periodo tanto en términos absolutos como relativos. A nivel espacial, se puede observar que la ciudad de Buenos Aires ha sido

\section{Gráfica 2}

Proporción de hogares con jefatura de Bolivia, Paraguay

y Perú con relación al total de hogares con jefatura extranjera.

Conurbano Bonaerense, 2001-2010

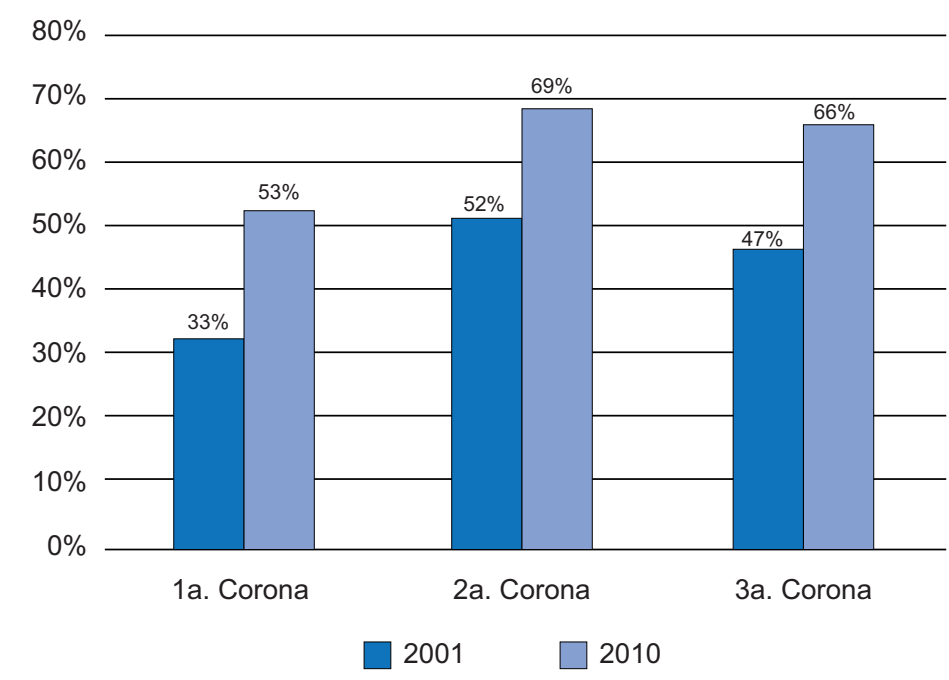

Fuente: Elaboración propia con base en datos del censo (INDEC, 2001, 2010). 
el territorio con el mayor crecimiento en términos de participación: este grupo pasó de $4.5 \%$ en 2001 a $7.2 \%$ en 2010 , lo que representa una variación relativa del $78 \%$ (Cuadro 4). Tal crecimiento fue algo menor en el resto de la región metropolitana (71\%). Por otro lado, en relación con los cordones, se observa que el Tercer Cordón ha sido aquel donde más han aumentado -en términos relativos- los hogares con jefes nacidos en Bolivia, Paraguay y Perú $(110 \%)$. No obstante, es necesario relativizar este dato debido al tamaño y la densidad de dicha población en el territorio.

En este sentido, se puede afirmar que, en principio, no se observan grandes diferencias en cuanto a una mayor concentración de los hogares con jefatura nacida en Bolivia, Paraguay y Perú según las tres envolventes (coronas) del conurbano. Es decir, la proporción de tales hogares es relativamente similar tanto en la ciudad de Buenos Aires como en la primera, segunda y tercera coronas.

Ahora bien, a nivel intrametropolitano, en el Mapa 3 se puede observar un claro patrón de asentamiento de dicha población en la RMBA. En efecto, en el año 2001 los territorios con la mayor proporción de hogares con jefatura nacida en Bolivia, Paraguay y Perú (entre 11.01 y 19.0\%) eran el Primer Cordón Oeste ${ }^{15}$ y el Segundo Cordón Sur ${ }^{16}$ del Conurbano Bonaerense. En la ciudad de Buenos Aires, por su parte, las zonas de mayor concentración de tales hogares (entre 5.1 y $11.0 \%$ ) eran Sur $2{ }^{17}$ y Centro. ${ }^{18}$ En este sentido, se advierte un patrón de asentamiento asociado a los corredores geográficos, en particular el Corredor Oeste y el Sur, los cuales presentan una mayor concentración de hogares con jefes/as de Bolivia, Paraguay y Perú en comparación con el Corredor Norte.

En el Mapa 4 se presenta la evolución de la población de Bolivia, Paraguay y Perú a nivel territorial. En primer lugar, cabe destacar que las zonas que concentraron el mayor crecimiento poblacional (entre 95 y $150 \%$ ) -en términos relativos- fueron el Tercer Cordón del Conurbano y la Zona Sur $1^{19}$ de la ciudad de Buenos Aires. En este marco, se puede afirmar la existencia de un patrón territorial de crecimiento muy claro focalizado principalmente en

15 El Primer Cordón Oeste corresponde a los municipios de Hurlingham, Morón, Ituzaingó y parte de La Matanza.

16 El Segundo Cordón Sur corresponde a los municipios de Ezeiza, Esteban Echevarría, Almirante Brown, Presidente Perón Florencio Varela y Berazategui.

17 La zona Sur 2 de la ciudad de Buenos Aires comprende los barrios de Liniers, Mataderos, Villa Lugano, Villa Riachuelo, Villa Soldati y Parque Avellaneda.

18 La zona Centro de la ciudad de Buenos Aires comprende los barrios de Barrio Parque, Recoleta, Balvanera, Monserrat, San Nicolás, Puerto Madero, San Telmo, San Cristóbal, Almagro y Boedo.

19 La zona Sur 1 comprende los barrios de Constitución, La Boca, Barracas, Parque Patricios y Nueva Pompeya. 


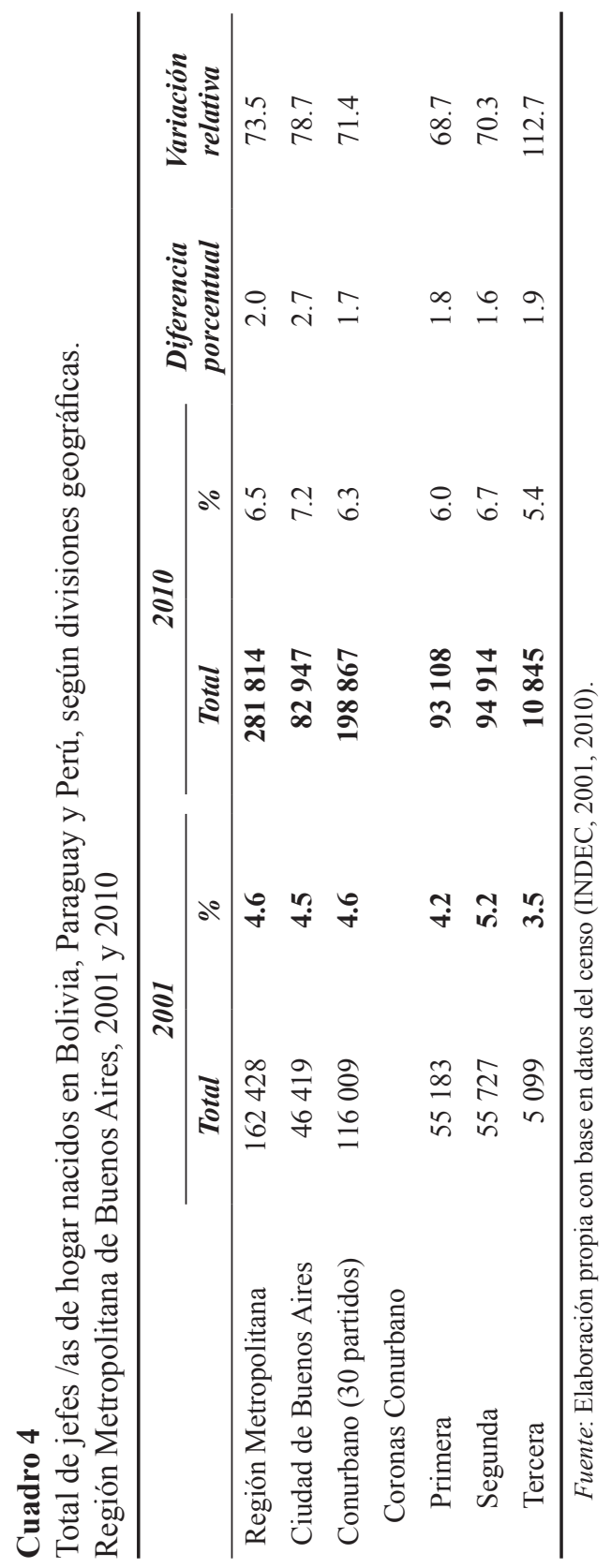




\section{Mapa 3}

Proporción de jefes/as de hogar nacidos/as en Bolivia, Paraguay y Perú. Región Metropolitana de Buenos Aires, 2001

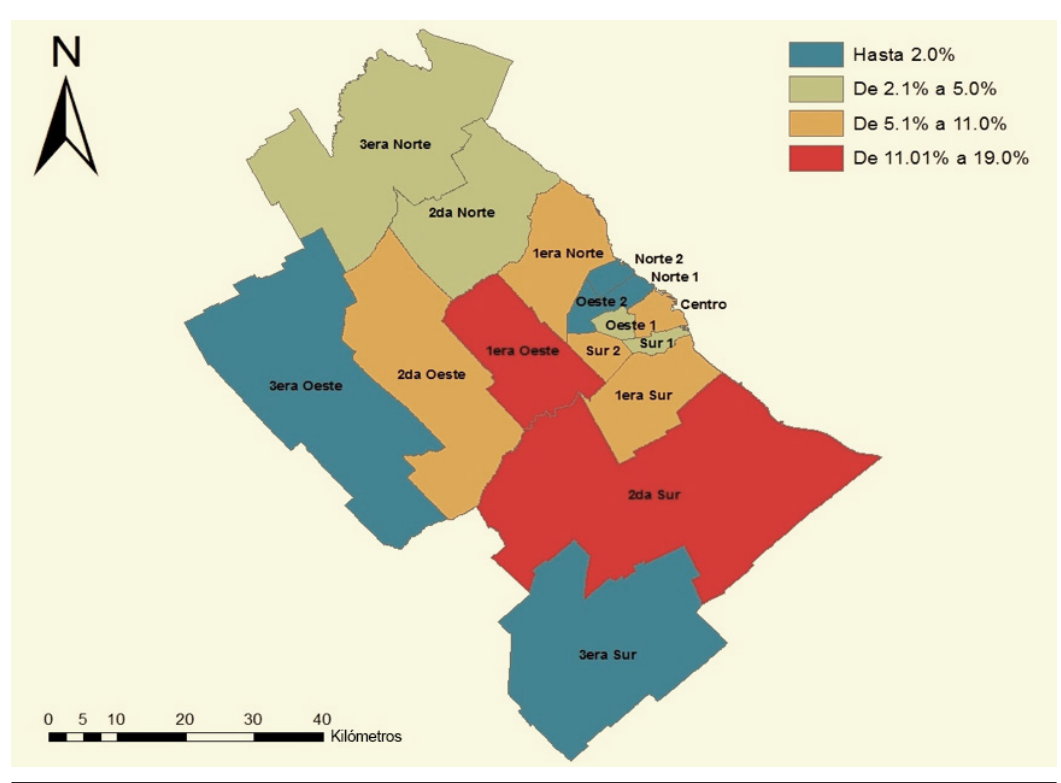

Fuente: Elaboración propia con base en datos del censo (INDEC, 2001, 2010).

el Corredor Oeste del Conurbano Bonaerense y en la zona Sur de la ciudad de Buenos Aires. Resultaría interesante enmarcar este movimiento con el crecimiento urbano que tuvo la RMBA durante el periodo 2001-2010 y, a su vez, analizarlo en el marco de las necesidades de vivienda de la población, en particular de los hogares emplazados en tales territorios. Sin embargo, debemos dejar planteados tales interrogantes para otras investigaciones, puesto que exceden los límites del presente trabajo.

\subsection{Distribución espacial de los hogares con jefatura extranjera (excluye Bolivia, Paraguay y Perú)}

Los hogares con jefes/as extranjeros/as (excluyendo a Bolivia, Paraguay y Perú) presentan otro patrón de asentamiento muy distinto. En principio, cabe destacar que, en lo referente al crecimiento poblacional durante el decenio, 


\section{Mapa 4}

Variación porcentual de jefes/as de hogar nacidos en Bolivia, Paraguay y Perú. Región Metropolitana de Buenos Aires, 2001-2010

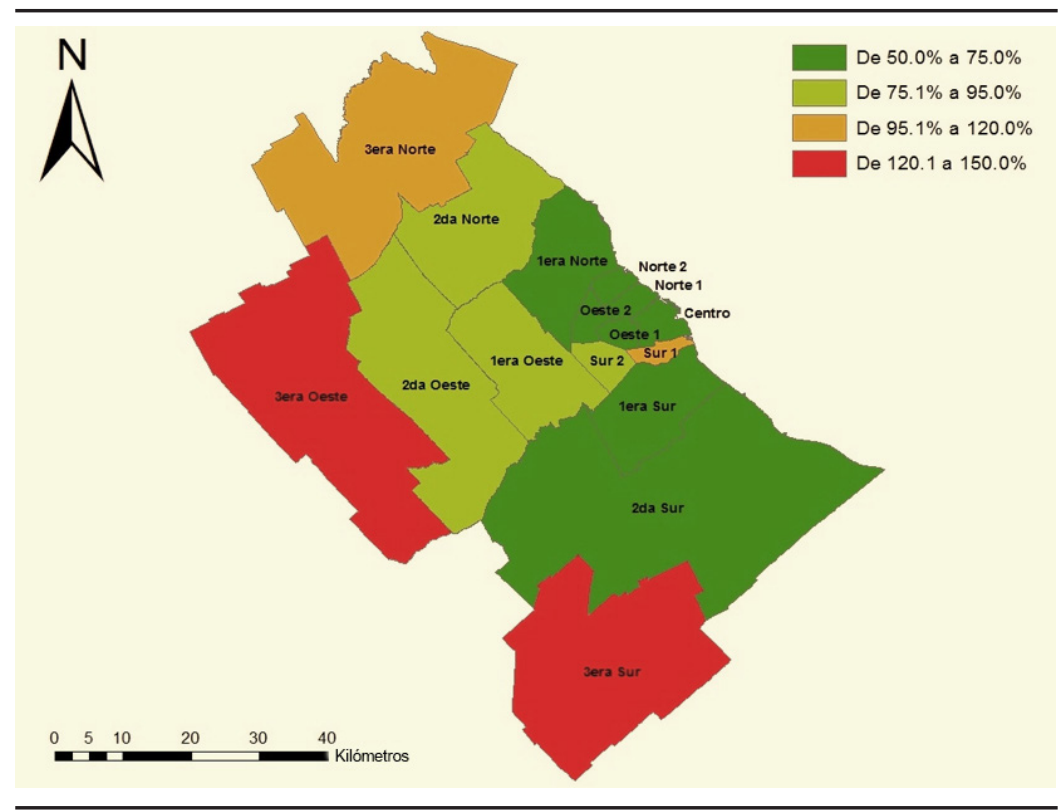

Fuente: Elaboración propia con base en datos del censo (INDEC, 2001, 2010).

dicha subpoblación presentó un signo negativo. Es decir, a nivel de la RMBA se produjo una contracción de tal población en términos relativos del $20 \%$. En el Cuadro 5 se advierte que el Conurbano Bonaerense ha sido el territorio en donde más se ha reducido (-22\%), a diferencia de la ciudad de Buenos Aires (-15\%). Esta disminución fue particularmente intensa en la Primera Corona, donde tales hogares pasaron del $8.6 \%$ en 2001 al 5.4\% en 2010, representando una reducción del 26 por ciento.

Cabe destacar que la distribución espacial de esta población aparece visiblemente asociada tanto a los cordones de la conurbación como a los corredores geográficos. Este patrón de asentamiento se caracteriza por una mayor concentración de hogares en el Primer Cordón y, en particular, en el Corredor Norte de la región metropolitana.

Así, en el Mapa 5 se puede apreciar una distribución espacial en la que determinadas zonas de la RMBA son las que concentran la mayoría de tales hogares. En 2001, los territorios con mayor proporción (entre 12.01 y 17.0\%) 


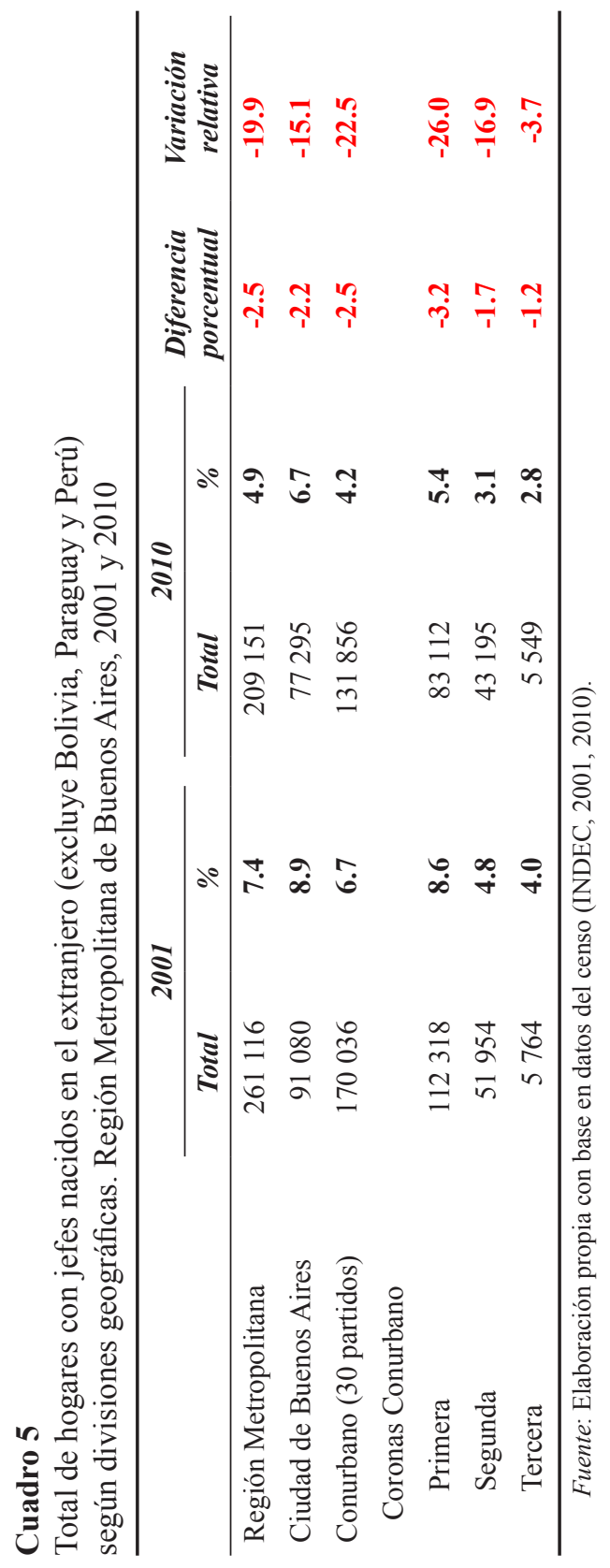




\section{Mapa 5}

Proporción de hogares con jefes nacidos en el extranjero (excluye Bolivia, Paraguay y Perú). Región Metropolitana de Buenos Aires, 2001

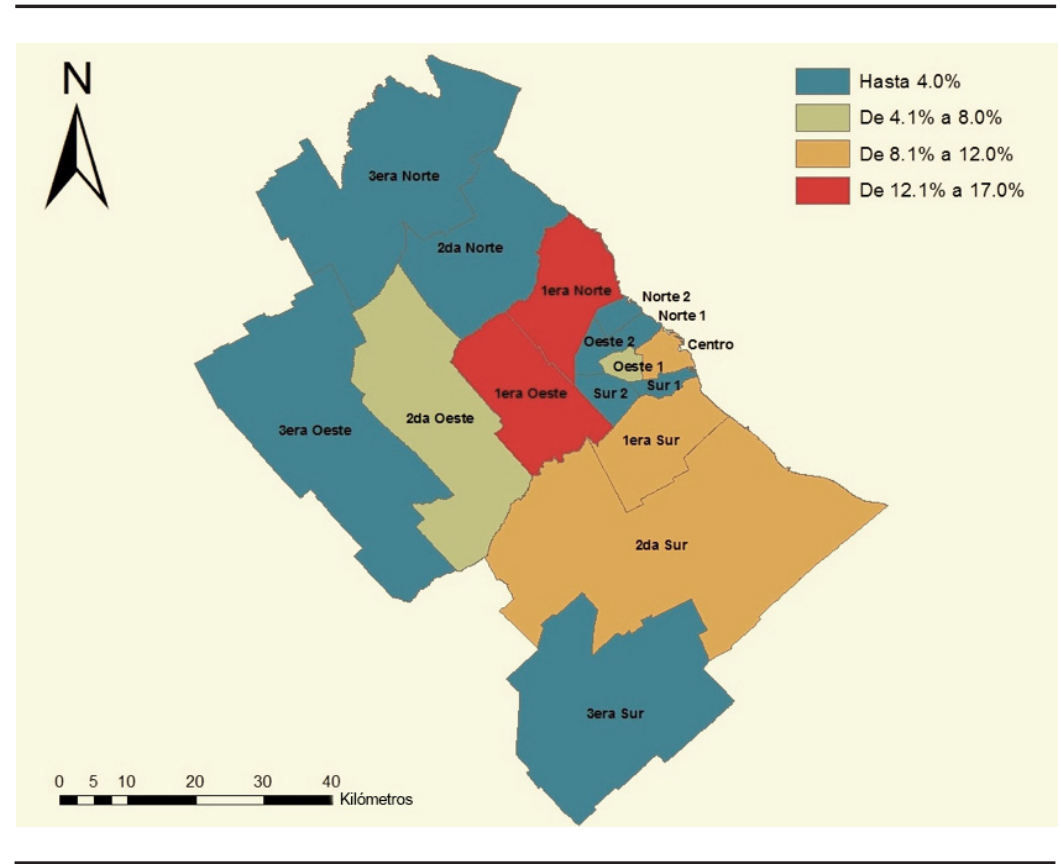

Fuente: Elaboración propia con base en datos del censo (INDEC, 2001, 2010).

eran el Primer Cordón Oeste y el Norte ${ }^{20}$ del Conurbano Bonaerense. Por su parte, en la ciudad de Buenos Aires, es la zona Centro aquella con mayor proporción (entre 8.1 y $12.0 \%$ ). En este sentido, resulta notorio que tanto en la periferia de la RMBA (Tercer Cordón) como en la periferia de la urbe (zona Sur 2, Oeste 2 y Norte 2) se encuentran la menores concentraciones y presencia (hasta 4 por ciento).

Por último, en el Mapa 6 se puede observar la evolución entre 2001 y 2010. Los territorios en donde más se redujo dicha población fueron el Primer Cordón Norte y el Primer Cordón Sur del Conurbano Bonaerense, y en el caso de la ciudad de Buenos Aires, la zona Sur 1 y la zona Oeste 2. En

20 El Primer Cordón Norte corresponde a los partidos-municipios de San Isidro, Vicente López, General San Martín y Tres de Febrero. 


\section{Mapa 6}

Variación porcentual de hogares con jefes nacidos en el extranjero (excluye Bolivia, Paraguay y Perú). Región Metropolitana de Buenos Aires, 2001-2010

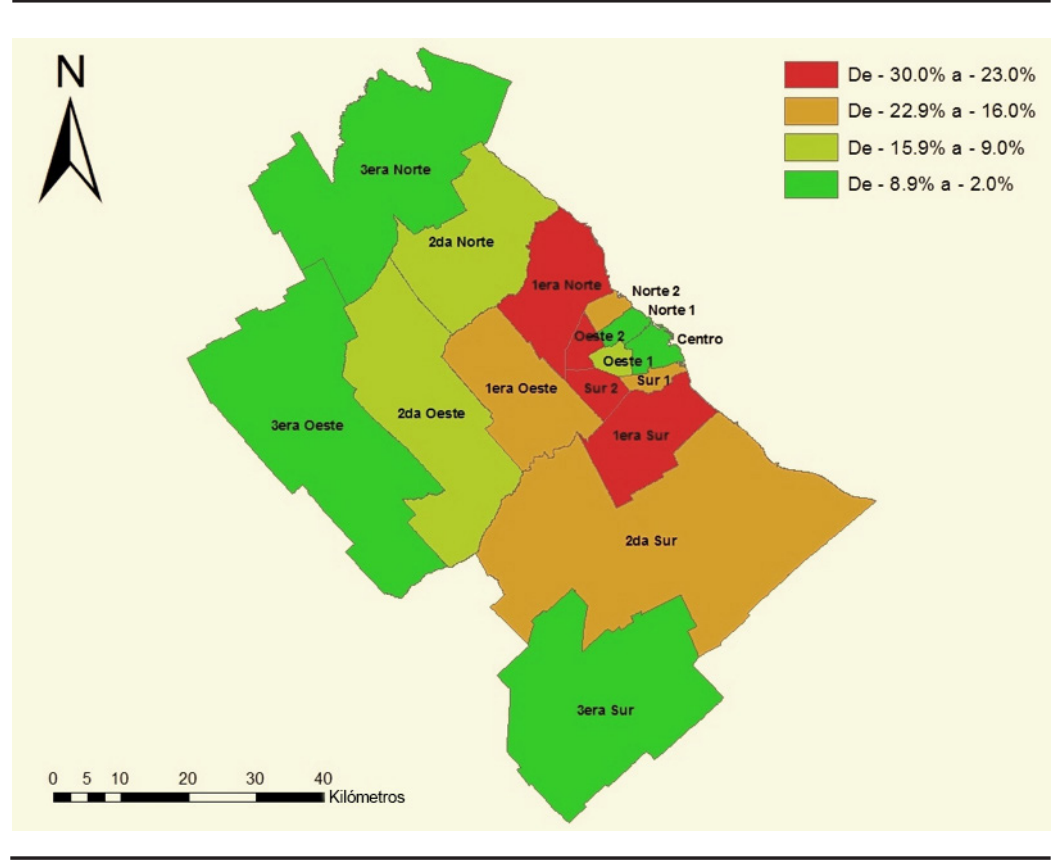

Fuente: Elaboración propia con base en datos del censo (INDEC, 2001, 2010).

tales territorios, la reducción fue entre 30 y $22 \%$. Asimismo, cabe advertir que, en el caso del Conurbano, la mayor reducción se produjo en uno de los territorios con mayor densidad de hogares inmigrantes (Primer Cordón Norte), mientras que en el caso de la urbe ocurrió en territorios en los que la presencia de dicha población es mucho menor. A diferencia del Conurbano Bonaerense, en la ciudad de Buenos Aires la zona de mayor densidad (Centro) fue en donde menos se ha reducido dicha población. 


\section{Conclusiones}

El artículo ha tenido como premisa general realizar aportes al conocimiento de los fenómenos migratorios en uno de los principales subsistemas migratorios de América Latina. En particular, se ha buscado profundizar el examen de los migrantes sudamericanos, en específico de los colectivos más dinámicos en la RMBA, en lo referente a su evolución reciente y sus patrones de distribución espacial a partir de un abordaje cuantitativo mediante técnicas de estadística espacial. De modo más general, se ha intentado también debatir sobre la presencia de procesos de segregación espacial hacia las comunidades migrantes en las ciudades de la región. Esta sección final, por tanto, se destinará a destacar de modo breve algunas conclusiones del análisis realizado.

En principio, cabe decir que la participación de los migrantes en el conjunto de la población de la RMBA hacia fines de 2010 continuó siendo relativamente baja tanto a nivel personas $(8.6 \%)$ como a nivel hogares (11.4\%). No obstante, debe destacarse que, en términos relativos, a nivel personas durante el decenio la población extranjera creció en $20 \%$, mientras que la población nativa en $6 \%$, ganando así la primera una mayor participación en el conjunto. A nivel hogares se registró un crecimiento en términos relativos del 15\%; sin embargo, este incremento resultó menor respecto al de los hogares nativos (22\%). En consecuencia, en términos de participación la población extranjera, a escala hogares, se mantuvo estable durante el periodo. Por consiguiente, la hipótesis de la ocurrencia de un crecimiento extraordinario de los flujos migratorios externos producto de una inusitada expansión económica debe descartarse. Ahora, si bien no se detectó un comportamiento extraordinario de los migrantes externos en relación con el conjunto de la población, no ocurrió lo mismo en el interior de la población inmigrante de la RMBA.

En esta dirección, el análisis efectuado con fuentes censales ha mostrado claras diferencias en la dinámica demográfica del colectivo de Bolivia, Paraguay y Perú en relación con los restantes grupos de inmigrantes. Dos hechos relevantes destacan al respecto: por un lado, el enorme incremento de dicho colectivo en relación con los restantes grupos de inmigrantes de Uruguay, Chile y Brasil; por otro, la fuerte reducción de los inmigrantes internacionales (excluyendo los de países limítrofes y Perú) registrada durante el periodo. En consecuencia, los flujos de migración externa dirigidos hacia la RMBA entre 2001 y 2010 fueron explicados en gran medida por los grupos de Bolivia, Paraguay y Perú; ahora bien, en términos de saldos migratorios, los incrementos en el stock de tales colectivos fueron compensados con las reducciones de los restantes colectivos de inmigrantes. Es así que, si 
bien a nivel global no se registró un crecimiento extraordinario -impulsado por un alza de la demanda de mano obra- dentro de la población inmigrante -como se demostró en el trabajo-, se produjo un importante cambio de composición durante el periodo, y este proceso, a nivel hogares, ha sido aún más marcado e intenso.

Por último, en lo referente a la distribución espacial del colectivo de Bolivia, Paraguay y Perú, el análisis socioespacial efectuado puso de manifiesto dos hallazgos relevantes. El primero es que estos grupos de inmigrantes se asientan de manera concentrada-segregada en determinadas zonas y territorios de la metrópoli, principalmente en aquellas caracterizadas por grandes déficits habitacionales y de infraestructura urbana (esto es, la zona Sur de la ciudad de Buenos Aires y el Primer Cordón Oeste y el Segundo Cordón Sur del Conurbano Bonaerense). El segundo es que el crecimiento demográfico intercensal del colectivo de Bolivia, Paraguay y Perú se produjo fundamentalmente en las zonas en las que se situaba dicha población en 2001. Por tanto, la distribución espacial de tipo concentrada-segregada de tal colectivo no sólo no se modificó, sino que se profundizó y reforzó durante el periodo. Por otra parte, los restantes grupos de migrantes presentaron claras diferencias con el colectivo de Bolivia, Paraguay y Perú con respecto a la distribución espacial, puesto que éstos se distribuyen principalmente en zonas de sectores medios y medios altos de la metrópoli (la zona Centro de la ciudad y en el Primer Cordón Oeste y el Norte del Conurbano Bonaerense). Es posible pensar entonces que, si bien el fenómeno de la segregación espacial en las ciudades de Latinoamérica se produce principalmente entre los grupos de niveles socioeconómicos polares, en los grupos migrantes -principalmente los que más crecieron en la RMBA- la acentuación de los patrones de distribución y asentamiento de tipo segregado puede estar dando cuenta de nuevas facetas en los procesos de segregación urbana, aspectos que deberán ser tratados y profundizados en nuevos estudios empíricos y futuras investigaciones.

\section{Bibliografía}

Balán, J. (1990). La economía doméstica y las diferencias entre sexos en las migraciones internacionales: un estudio sobre el caso de los bolivianos en la Argentina. Estudios Migratorios Latinoamericanos, 15-16, 269-294.

Benencia, R. (1997). De peones a patrones quinteros. Movilidad social de familias bolivianas en la periferia bonaerense. Estudios Migratorios Latinoamericanos, 12(35), 63-102. 
Benencia, R. (2003). La inmigración limítrofe. En F. Devoto (coord.), Historia de la inmigración en la Argentina (pp. 433-484). Buenos Aires, Argentina: Sudamericana.

Bruno, S. (2007). Cifras imaginarias de la inmigración limitrofe en la Argentina. Ponencia presentada en las VII Jornadas de Sociología. Universidad de Buenos Aires, Argentina, noviembre.

Burgess, E. (1925). The growth of city: An introduction to a research project. En R. Park, E. Burgess y R. McKenzie (coords.), The city (pp. 47-62). Chicago, IL: The University of Chicago Press.

Buzai, G., Baxendale, C., Rodríguez, L. y Escanes, V. (2003). Distribución y segregación espacial de los extranjeros en la ciudad de Luján. Un análisis desde la geografía cuantitativa. Signos Universitarios, 22(39), 29-53. Recuperado de https://p3.usal.edu.ar/index.php/signos/article/ view/2229/2777

Castells, M. (1979). La cuestión urbana. Madrid, España: Siglo XXI.

Cerrutti, M. (2005). La migración peruana a la ciudad de Buenos Aires: su evolución y características, Población de Buenos Aires 2(2), 7-28. Recuperado de http://www.redalyc.org/pdf/740/74020201.pdf

Cerrutti, M. (2009). Diágnostico de la poblaciones de inmigrantes en la Argentina (Documentos de la Dirección Nacional de Población, núm. 2). Buenos Aires: Ministerio del Interior de la República Argentina.

Cerruti, M. y Maguid, A. (2007). Inserción laboral e ingresos de migrantes limítrofes y del Perú en el Área Metropolitana de Buenos Aires, 2005 (Notas de Población, núm. 83). Santiago de Chile: Comisión Económica para América Latina y el Caribe.

Cerruti, M. y Parrado, E. (2001). Migración laboral de paraguayos en la Argentina: entrada a los mercados de trabajo y trayectorias ocupacionales. Estudios Migratorios Latinoamericanos, 16 (48) 369-399.

Ceva, M. (2006). La migración limítrofe hacia la Argentina en la larga duración. En A. Grimson y E. Jelin (coords.), Migraciones regionales hacia la Argentina. Diferencia, desigualdad y derechos (pp. 17-46). Buenos Aires, Argentina: Prometeo.

Chavez Galindo, A., Rodríguez Vignoli, J., Acuña, M., Barquero, J., Macadar, D., Pinto da Cunha, J. y Sobrino, J. (2016). Migración interna y cambios metropolitanos: ¿qué esta pasando en las grandes ciudades de América Latina? Revista Latinoamericana de Población, 10(18), 7-41. Recuperado de http://biblioteca.ccp.ucr.ac.cr/bitstream/handle/123456789/1316/ Barquero.pdf? sequence $=1$ \&isAllowed $=\mathrm{y}$

Cortés, M. y Groisman, F. (2004). Migraciones, mercado de trabajo y pobreza en el Gran Buenos Aires. Revista de la CEPAL, 82, 173-191. Recu- 
perado de https://repositorio.cepal.org/bitstream/handle/11362/109 47/082173191_es.pdf?sequence $=1$

Darroch G. y Marston W. (1971). Social class basis of ethnic residential segregation: The Canadian case. The American Journal of Sociology, 3, 491-510. Recuperado de https://www.journals.uchicago.edu/doi/ $10.1086 / 225160$

Devoto, F. (2003). Historia de la inmigración en la Argentina. Buenos Aires, Argentina: Sudamericana.

Garay, A. (2007). Lineamientos estratégicos para la Región Metropolitana de Buenos Aires. Gobierno de la Provincia de Buenos Aires, Subsecretaría de Urbanismo y Vivienda.

Gómez, P. y Sánchez Soria, D. (2017). Cambios y continuidades en la incorporación espacial de los migrantes peruanos en la ciudad de Córdoba, Argentina. Remhu, Revista Interdisciplinar da Mobilidade Humana, 25(30), 133-156. Recuperado de http://www.scielo.br/pdf/remhu/ v25n50/1980-8585-remhu-25-50-133.pdf

Harvey, D. (1997). Urbanismo y desigualdad social. Madrid, España: Siglo XXI.

Instituto Nacional de Estadísticas y Censos (INDEC). (1997). La migración internacional en la Argentina: sus características e impactos (Estudios, núm. 29). Buenos Aires, Argentina: Instituto Nacional de Estadísticas y Censos.

Instituto Nacional de Estadísticas y Censos (INDEC). (2001). Censo Nacional de Población, Hogares y Viviendas del año 2001. Buenos Aires, Argentina: Instituto Nacional de Estadísticas y Censos.

Instituto Nacional de Estadísticas y Censos (INDEC). (2007). Encuesta Complementaria de Migraciones Internacionales 2002-2003 (Base de datos). Buenos Aires, Argentina: Instituto Nacional de Estadísticas y Censos.

Instituto Nacional de Estadísticas y Censos (INDEC). (2010). Censo Nacional de Población, Hogares y Viviendas del año 2010. Buenos Aires, Argentina: Instituto Nacional de Estadísticas y Censos.

Leerkes, A., Engbersen, G. y San, M. (2007). Shadow places: Patterns of spatial concentration and incorporation of irregular immigrants in the Netherlands. Urban Studies, 44(8), 1491-1516. Recuperado de https:// journals.sagepub.com/doi/pdf/10.1080/00420980701373479

Linares, S. (2013). Medidas de segregación socioespacial: discusión metodológica y aplicación empírica sobre ciudades medias argentinas. Persona y Sociedad, 27(2), 11-40. Recuperado de https://core.ac.uk/down load/pdf/52476850.pdf 
Maguid, A. (2008). Políticas migratorias y fronteras socioeconómicas en el Cono Sur. En A. Pécoud y P. de Guchteneire (eds.), Migración sin fronteras. Ensayos sobre la libre circulación de las personas (pp. 341-364). París, Francia: UNESCO (Estudios Sociales).

Maguid, A. y Arruñada, V. (2005). El impacto de la crisis en la inmigración limítrofe y del Perú hacia el Área Metropolitana de Buenos Aires. Estudios del Trabajo, 30, 95-122. Recuperado de https://www.aset.org.ar/ docs/Maguid\%20Arrunada\%2030.pdf

Maguid, A. y Bruno, S. (2010). Migración, mercado de trabajo y movilidad ocupacional: el caso de los bolivianos y paraguayos en el Área Metropolitana de Buenos Aires. Población de Buenos Aires, 7(12), 7-28. Recuperado de http://www.redalyc.org/html/740/74015656001/

Marcos, M. y Mera, G. (2015a). Migrantes internacionales en la Aglomeración Gran Buenos Aires: un análisis cuantitativo de su distribución espacial. Cuadernos Geográficos, 54(1), 257-282. Recuperado de http:// revistaseug.ugr.es/index.php/cuadgeo/article/view/2581/3235

Marcos, M. y Mera, G. (2015b). Cartografías migratorias urbanas. Distribución espacial de la población extranjera en la Ciudad de Buenos Aires (2010). Geograficando. 11(1). Recuperado de https://dialnet.unirioja.es/ servlet/articulo?codigo $=5160037$

Mármora, L. (2002). Las políticas de migraciones internacionales. Buenos Aires, Argentina: Paidós.

Marshall, A. (1979). Inmigrant workers in the Buenos Aires labor market. The International Migration Review, 13(3), 488-501. Recuperado de https://www.jstor.org/stable/2545578?seq=1\#page_scan_tab_contents

Marshall, A. (1983). Inmigración de países limítrofes y demanda de mano de obra en la Argentina 1940-1980. Desarrollo Económico, 23(89), 3558. Recuperado https://www.jstor.org/stable/3466446?seq=1\#page_ scan_tab_contents

Marshall, A. y Orlansky, D. (1981). Las condiciones de expulsión en la determinación del proceso emigratorio desde países limítrofes hacia la Argentina. Desarrollo Económico, 20(80), 491-510. Recuperado de https://www.jstor.org/stable/3466715?seq=1\#page_scan_tab_contents

Martínez Pizarro, J. (2003). El mapa migratorio de América Latina y el Caribe, las mujeres y el género (Población y Desarrollo, núm. 44). Santiago de Chile: Comisión Económica para América Latina y el Caribe.

Matossian, B. (2010). Migración chilena y segregación urbana: el caso de San Carlos de Bariloche (Tesis doctoral, Universidad Nacional de Cuyo, Argentina). 
McKenzie, R. (1925). The ecological approach to the study of the human community. En R. Park, E. Burgges y R. Mckenzie (coords.), The city (pp. 63-73). Chicago, IL: The University Chicago Press

Meichtry, N. y Beck, H. (2002). Caracterización demográfica de la inmigración limítrofe en el nordeste de Argentina en la primera mitad del siglo XX. En H. Asdrúbal Silva (ed.), Historia de las migraciones limítrofes en el Cono Sur de América, Argentina, Bolivia, Brasil, Chile, Paraguay y Uruguay. Primera parte del siglo XIX a 1960 (pp. 145-186). Ciudad de México: Instituto Panamericano de Geografía e Historia.

Mera, G. (2014). Migración paraguaya en la Ciudad de Buenos Aires (2010): distribución espacial y pobreza. Revista Latinoamericana de Población, 14, 57-80. Recuperado de http://revistarelap.org/ojs/index.php/relap/ article/view/18/20

Mera, G. y Vaccoti, L. (2013). Migración y déficit habitacional en la Ciudad de Buenos Aires: resignificando el problema. Argumentos. Revista de Crítica Social, 15, 176-202. Recuperado de http://publicaciones.sociales. uba.ar/index.php/argumentos/article/view/906/792

Mignone, A. (2010). La segregación residencial de los migrantes paraguayos en las ciudades de Formosa y Posadas. Ponencia presentada en el V Taller Paraguay desde las Ciencias Sociales, Asunción, Paraguay.

Molinatti, F. (2013). Segregación residencial socioeconómica en la ciudad de Córdoba (Argentina): tendencias y patrones espaciales. Revista INVI, 28(79), 61-94. Recuperado de http://www.revistainvi.uchile.cl/index. php/INVI/article/view/778/1116

Molinatti, F. y Peláez, E. (2014). Segregación residencial y migrantes de Bolivia y del Perú. Niveles y patrones espaciales en la ciudad de Córdoba a comienzos del siglo XX. Ponencia presentada en el VI Congreso de la Asociación Latinoamericana de Población, Lima, Perú.

Otero, H. y Pellegrino, A. (2004). Compartir la ciudad. Patrones de residencia e integración de inmigrantes en Buenos Aires y Montevideo durante la inmigración masiva. En H. Otero (dir.), El mosaico argentino. Modelos y representaciones del espacio y de la población, siglos XIX-XX (pp. 19-69). Buenos Aires, Argentina: Siglo XXI.

Park, R. (1926). The urban community as a spatial pattern and a moral order. En E. Burgess (ed.), The urban community: Selected papers from the proceedings of the American Sociological Society, 1925 (pp. 3-18). Chicago, IL: The University of Chicago Press.

Parrado, E. y Cerrutti, M. (2003). Labor migration between developing countries: The case of Paraguay and Argentina. International Migration 
Review, 37(1), 101-132. Recuperado de https://journals.sagepub.com/ doi/pdf/10.1111/j.1747-7379.2003.tb00131.x

Perren, J. (2010). Estructura urbana, mercado laboral y migraciones: una aproximación al fenómeno de la segregación en una ciudad de la Patagonia (Neuquén: 1960-1990). Miradas en Movimiento, 4, 36-69. Recuperado de https://dialnet.unirioja.es/servlet/articulo? $\operatorname{codigo}=3307800$

Portes, A. (1998). Social capital: Its origins and applications in modern sociology. Annual Review of Sociology, 24(1), 1-24. Recuperado de https:// www.annualreviews.org/doi/pdf/10.1146/annurev.soc.24.1.1

Rodríguez, J. y Arriagada, C. (2004). Segregación residencial en la ciudad latinoamericana. Eure, Revista Latinomericana de Estudios Urbano Regionales 30(89), 5-24. Recuperado de https://scielo.conicyt.cl/scielo. php?script $=$ sci_arttext\&pid $=$ S0250-71612004008900001

Rosas, C. (2008). Antes de migrar: aspectos sociodemográficos, género y redes en la migración peruana en Buenos Aires. Debates en Sociología, 33. Recuperado de http://revistas.pucp.edu.pe/index.php/debatesenso ciologia/article/view/2541/2485

Rosas, C. (2010). Implicaciones mutuas entre el género y la migración. Mujeres y varones peruanos. Buenos Aires, Argentina: Eudeba.

Sassone, S. y De Marco, G. (1994). Problemáticas territoriales, asentamientos y dinámica de la inmigración limítrofe. Geodemos, 2, 179-298.

Sassone, S. González, M. y Matossian, B. (2010). Urbanización e inmigración limitrofe en la Argentina: casos de homogeneidad y diversidad migratoria. Trabajo presentado en el Seminario Internacional sobre Población y Sociedad en América Latina, Salta, Argentina.

Schteingart, M. y Torres, H. (1973). Procesos sociales y estructuración metropolitana en América Latina. Estudio de casos. Desarrollo Económico, 12(48), 725-760. Recuperado de https://www.jstor.org/stable/3466 302 ?seq=1\#page_scan_tab_contents

Torres, H. (2001). Cambios socioterritoriales en Buenos Aires durante la década de 1990. Eure, Revista Latinoamerica de Estudios Urbano Regionales, 27(80), 33-56. Recuperado de http://www.eure.cl/index.php/ eure/article/view/1230

Villaça, F. (1998). Espaço intra-urbano no Brasil. São Paulo, Brasil: Studio Nobel. 


\section{Acerca del autor}

Albano Blas Vergara Parra es sociólogo y maestro en Historia Económica y de la Políticas Económicas por la Universidad de Buenos Aires, y doctor en Ciencias Sociales por la Facultad Latinoamericana en Ciencias Sociales. Es profesor en la Facultad de Ciencias Sociales de la Universidad de Buenos Aires. Ha publicado artículos en revistas científicas nacionales e internacionales, entre ellos:

Vergara Parra, A. (2018). Segregación socioterritorial en la Región Metropolitana de Buenos Aires: análisis espacial intraurbano, características y evolución reciente (2001-2010). Notas de Población, 106, 251-284. Santiago de Chile: Comisión Económica para América Latina y el Caribe.

Vergara Parra, A. (2018). Controversias y aportes en torno a la cuantificación de la población en villas de emergencia y asentamientos precarios en la Región Metropolitana de Buenos Aires (RMBA). Período 2001-2010. Revista Iberoamericana de Urbanismo, 14, 87-103.

Vergara Parra, A. (2017). El problema habitacional y su evolución reciente en la Región Metropolitana Buenos Aires: avances y retrocesos entre 2001 y 2010. Revista Latinoamericana de Población, 21(11), 7-32.

Recepción: 4 de septiembre de 2017.

Aceptación: 11 de abril de 2018. 
